\title{
Mixed burst and tonic firing in the thalamus: A study in the feline lateral geniculate nucleus in vivo
}

\author{
Kenneth L. Grieve, Casto Rivadulla, Javier Cudeiro
}

\begin{abstract}
Compounds known to inhibit or disfacilitate cells in cat dorsal lateral geniculate nucleus (dLGN) were applied iontophoretically in vivo. Application of GABA, or agonists of $\mathrm{GABA}_{\mathrm{A}}$ and $\mathrm{GABA}_{\mathrm{B}}$ receptors, markedly decreased responses to low frequency periodic visual stimulation, but, while causing some increases in burst firing, cells continued to produce tonic spikes even when firing was reduced to near zero. Similar actions were seen with compounds manipulating the cholinergic system. Inhibition of local Nitric Oxide production reduced firing rates but did not affect burst firing. Significant levels of tonic firing were found mixed with burst firing throughout the recordings even under conditions most favourable for bursting. We suggest that the local synaptic input to an individual dLGN cell is sufficiently dynamic to prevent the prolonged periods of burst firing which can be evoked in brain slice preparations.
\end{abstract}

Keywords. Thalamus; Cat; GABA; Nitric oxide; Acetylcholine

\section{Introduction}

It has been known for some time that thalamic cells have the intrinsic property of a bi-stable firing pattern — known as “tonic" and "burst” firing modes (Jahnsen and Llinas, 1984a and Jahnsen and Llinas, 1984b). During tonic firing the cell responds to afferent input with an output which is related to the intensity of its input. During burst mode, the cell's response is very non-linear, giving rise to a high frequency burst of action potentials, essentially decoupled from the stimulus parameters. While this second mode has classically been associated with sleep (Glen and Steriade, 1982; for a review see Llinas and Steriade, 2006), evidence suggests not only that a proportion of cells fire in bursts in the wake state (Guido and Weyand, 1995 and Ramcharan et al., 2000), but these bursts may serve a specific function, as a wake-up call to target cortical cells (for a review see Sherman, 2005). Bursts are known to occur when a cell is hyperpolarised for a sufficient period to de-inactivate a voltage dependent $\mathrm{Ca}^{++}$channel - at which point a depolarising stimulus will trigger a calcium spike, crowned by the high frequency burst of sodium/potassium mediated action potentials (Jahnsen and Llinas, 1984b). However, as originally described, at the end of the burst, the cell is once more hyperpolarised and will therefore continue to respond in bursts to its afferent input, (provided the input is of a relatively low temporal frequency, McCormick and Feeser, 1990). This is the pattern of events which has been reported in the literature in studies involving brain slice preparations (Crunelli et al., 1988 and Soltesz et al., 1989), in which stable current injection can actively control membrane polarity levels (McCormick and Pape, 1990). However, it is clear that in vivo such stable conditions do not exist and a "middle state" of mixed burst and tonic firing may exist with cells switching "modes" continuously ( Guido et al., 1995); a result of the delicate balance of currents, including the concept of "windowed" currents ( Crunelli et al., 2005) which operate over a range of membrane polarities, rather than an abrupt shift at a single voltage level.

Thus the evidence shows that the "instant" firing state of thalamic relay cells may be more dependent upon the mixture of synaptic input, both from visual structures like retina and cortex, as well as brainstem afferents, all acting together on thalamic mechanisms which, critically, include significant local inhibition (McCormick and Pape, 1988, McCormick and Bal, 1994, Pape and McCormick, 1995 and Wolfart et al., 2005). It has most recently been suggested that local GABAergic inhibition may be a requirement for the generation of burst; as withdrawal of excitation may be ineffective (Wang et al., 2007).

In these experiments we asked a simple question: Can we artificially shift cells from tonic to burst firing mode in vivo by application of substances known to hyperpolarise cells or mimic the actions of 
the inhibitory neurotransmitter GABA, to induce a state of continuous burst responsiveness, and would we see an abrupt shift (as demonstrated in vitro)? Further, were substances acting via modulation of the GABA system more effective than those operating via other neurotransmitters?

\section{Results}

We examined 48 cells including $\mathrm{ON}$ and OFF and $\mathrm{X}$ and $\mathrm{Y}$ subtypes. We could find no obvious differences between these groups with respect to the results described here and so we have considered the population as a whole.

\subsection{Application of $G A B A$}

Application of GABA was tested on 13 cells and the simple method is exemplified in Figs. 1A, B. The protocol was as follows: establish a base-line visual response magnitude, and repeat the stimulation during increasing levels of application of GABA. As shown in Fig. 1B, the magnitude of the visual response clearly decreased as the application of GABA was stepped up from control levels, through a reduction in backing current, to full iontophoretic ejection at current levels up to $60 \mathrm{nA}$. There was a near linear reduction in firing rate in response to the visual stimulus as GABA application was increased in intensity. At the last test point the cell fired $<\sim 10 \%$ of its control level, yet the proportion of these included in bursts was only $14 \%$.

A
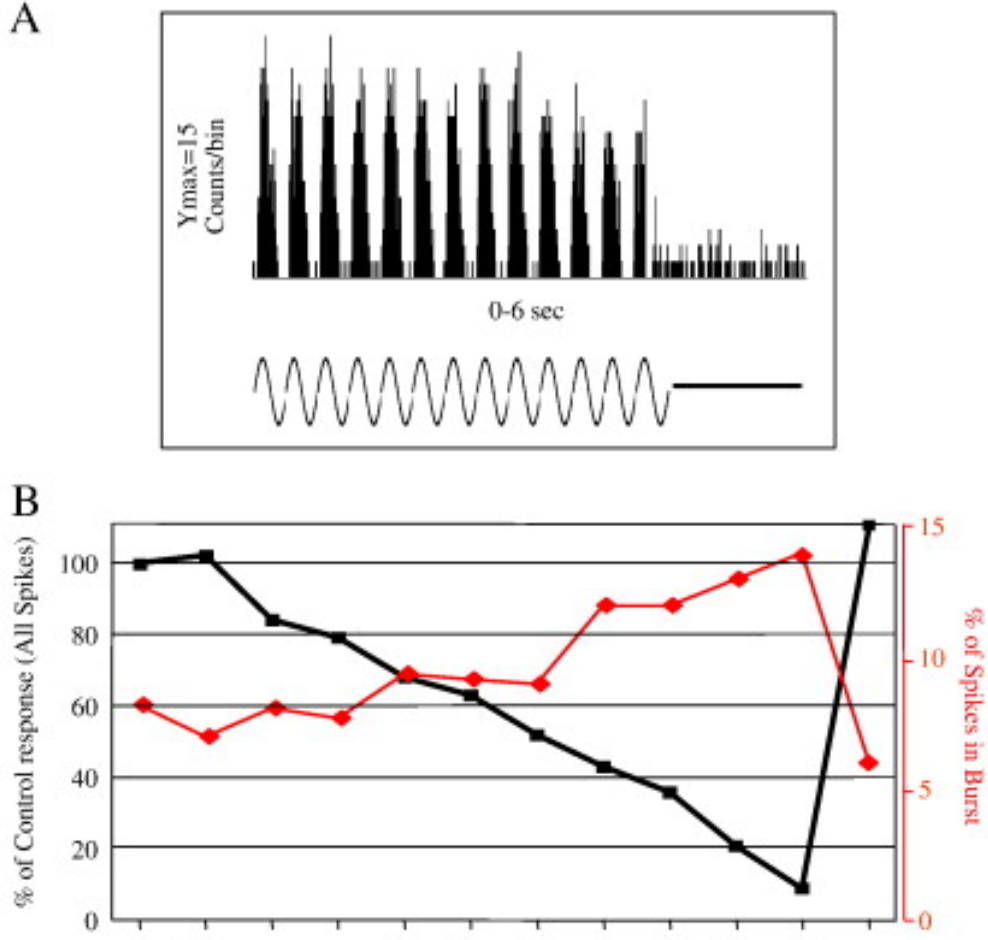

Number of spikes

Tonic mode

Burst mode

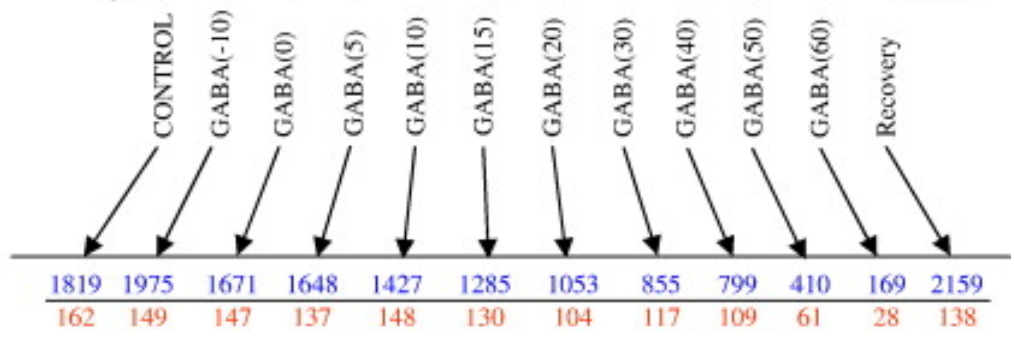

Fig. 1.- (A) Representative PSTH indicating the visual responsiveness of a cell under control conditions. This represents a period of visual activation with the sinusoidal grating and a subsequent period of unstimulated activity. The visual stimulus was a drifting grating of 0.3 cycles $/{ }^{\circ}$ presented at 3 cycles/s. This pattern was repeated and averaged to produce the PSTH (B) The Histogram representation of the firing levels (black line, left axis) of an ON, Y cell, in control conditions and during iontophoretic application of GABA. The \% of the spikes which occur in bursts is also shown (red line, right axis). The firing pattern of the cell is shown in the peri-stimulus time histogram (PSTH) above the histogram. 
In another example, shown in Fig. 2, we continued the GABA application until the cell neared silence. In this case, the proportion of spikes occurring in bursts increased as GABA ejection was raised to + $5 \mathrm{nA}$ (from 18\% to $27 \%$ ). Using GABA we were unable (under any conditions) to affect a change of "mode" such that a cell fired only in bursts - in all cases, burst firing remained mixed with tonic spikes. In this example, subsequent application of ACh significantly increased the visual response magnitude above control levels, and reduced the proportion of spikes occurring in bursts. The lowest level of bursting we achieved (+ $50 \mathrm{nA} \mathrm{ACh)} \mathrm{in} \mathrm{this} \mathrm{case} \mathrm{was} 12 \%$ (see below for more details on the effect of cholinergic manipulation).

A
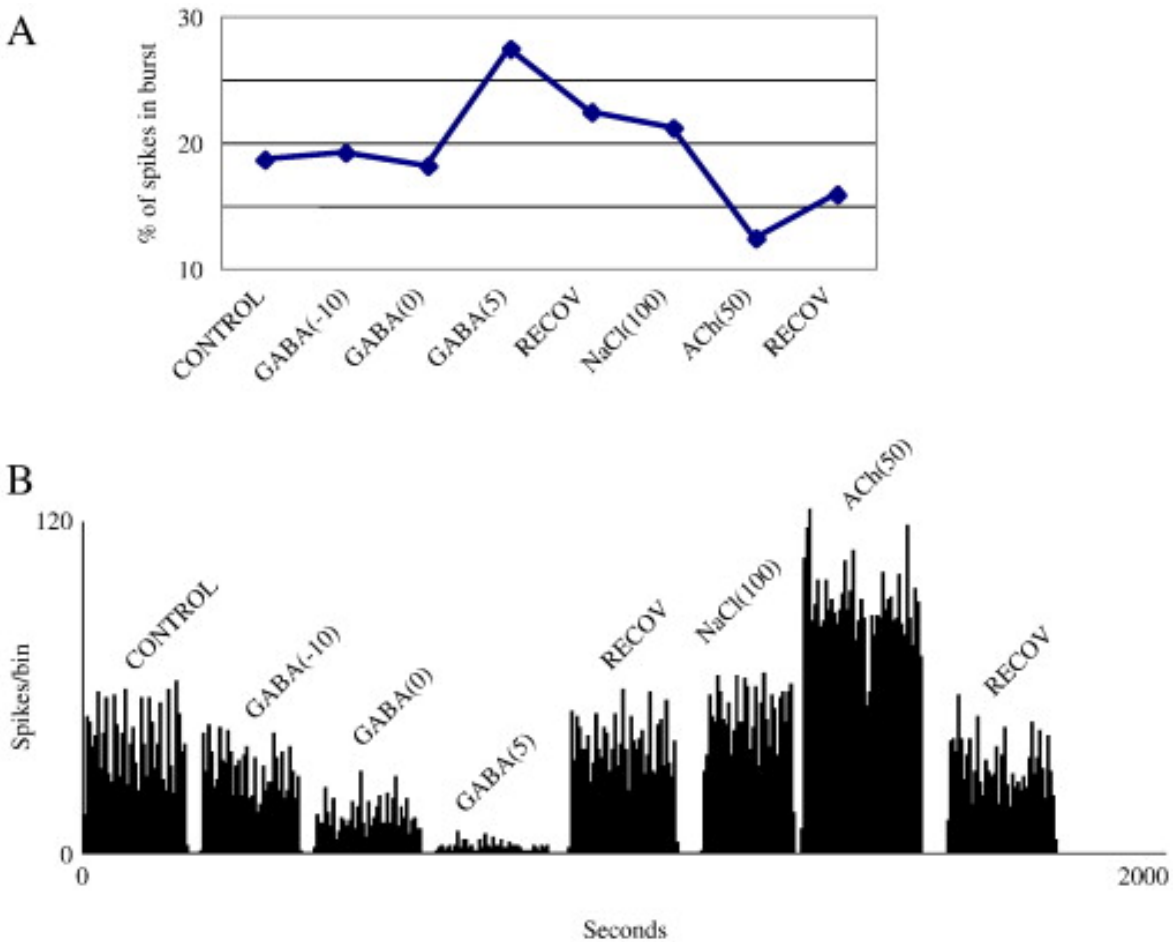

Fig. 2. - (A) \% of spikes fired in bursts for an Y, OFF cell treated with increasing levels of GABA, from control conditions, in which GABA is retained by $-25 \mathrm{nA}$, through GABA at $-10,0$ and $+5 \mathrm{nA}$ of application. The cell is then "recovered" with the GABA retention set again to $-25 \mathrm{nA}$. Following this we applied $+100 \mathrm{nA}$ of current through a barrel containing $0.9 \% \mathrm{NaCl}$, to mimic the effect of current application - no change in firing was seen. Finally we applied $+50 \mathrm{nA}$ of ACh which decreased the level of burst firing and increased visual responsiveness (see below). (B) Actual PSTH for the visual responses of the cell before during and after drug application. Note that each "block" of firing represents the response to repeated visual stimulation with a drifting grating of appropriate spatial frequency. Visual stimulation was stopped between drug level changes. The entire recording took $\sim 30 \mathrm{~min}$ and is typical of the duration for this type of experiment. Data is shown in $5 \mathrm{~s}$ bins. The visual stimulus was a drifting

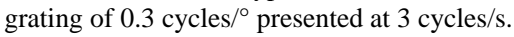

We also tested agonist compounds selective for the two main types of GABA receptor, isoguvacine for $\mathrm{GABA}_{\mathrm{A}}(n=5)$ and baclofen for $\mathrm{GABA}_{\mathrm{B}}(n=12)$. Both compounds reduced cell firing. This is illustrated in Fig. 3 and Fig. 4, showing the actions of isoguvacine and baclofen respectively. Isoguvacine, like GABA, reduced cell firing and could increase the \% of spikes fired in bursts, but, also like GABA, never produced a state of continuous bursting. In the example of baclofen action, the compound first increased the $\%$ of spikes found in bursts (from 2\% to 14\%), while decreasing the total number of spikes fired. However, increased application current from 70 to $90 \mathrm{nA}$ resulted in a further decrease in spike activity but a concomitant decrease in the \% of spikes found to be in bursts. Typically, however, both isoguvacine and baclofen applications increased the number of spikes fired in bursts, as the total number of spikes in the visual response decreased. Crucially, however, as with GABA, the burst firing was always accompanied by tonic spikes. 


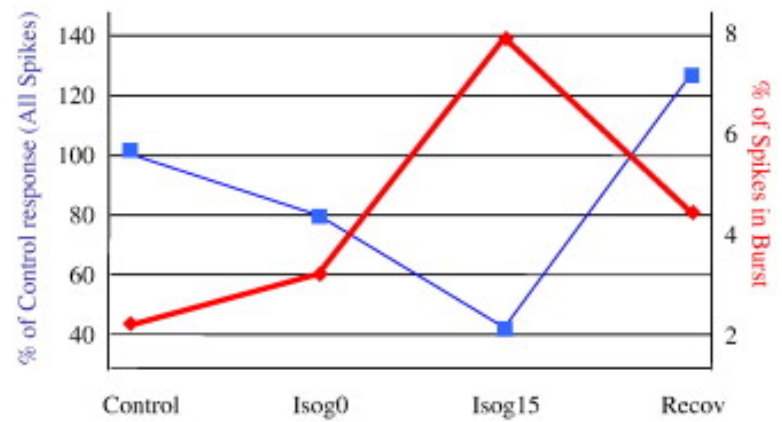

Fig. 3. - Example of the effect of application of the $\mathrm{GABA}_{\mathrm{A}}$ agonist isoguvacine. Like GABA itself, the substance significantly decreased firing and increased the \% of spikes in the response which were within bursts, but never achieved a shift to complete burst "mode" firing. The maximum $\%$ seen was $\sim 8 \%$.

A
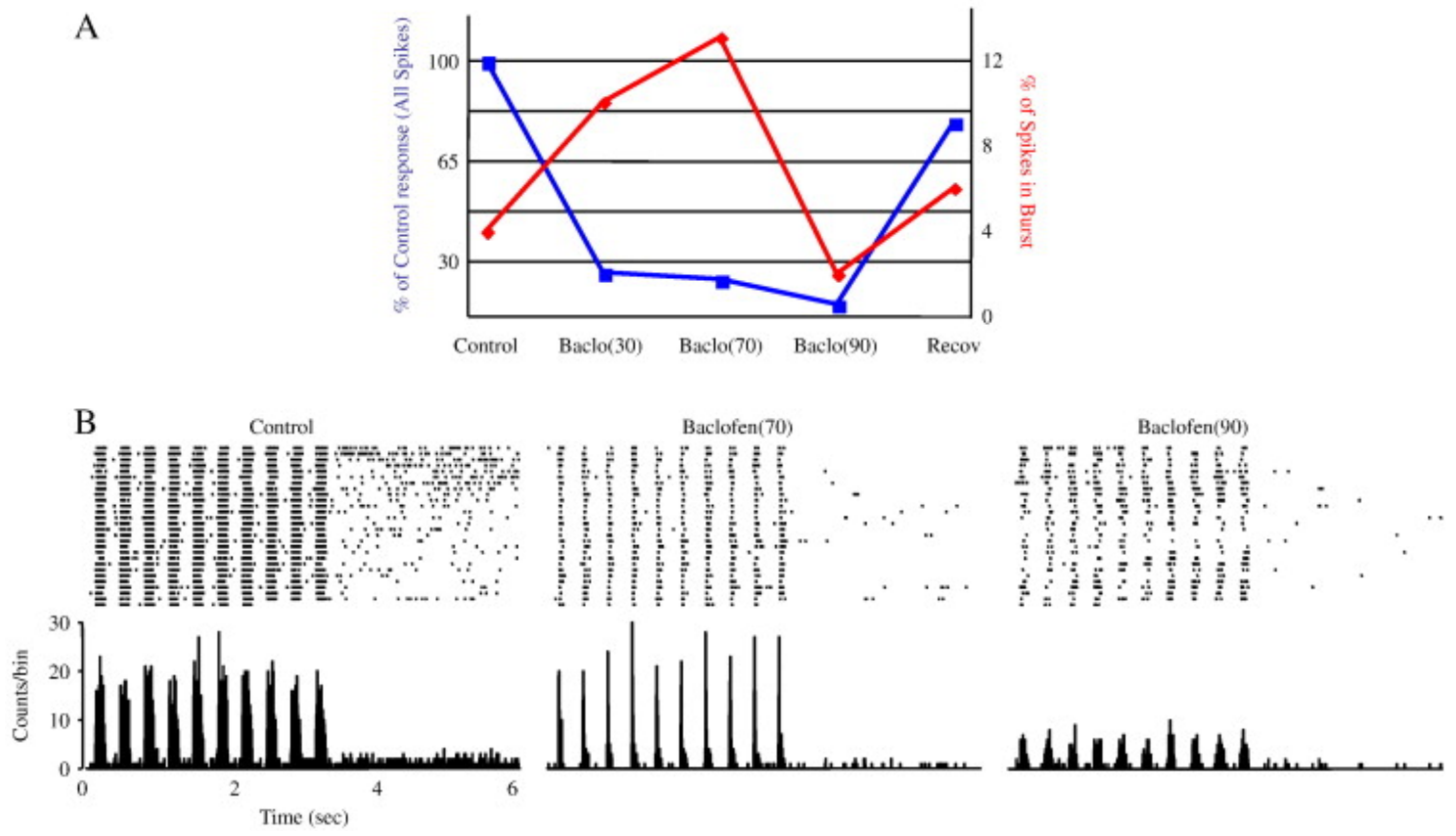

Fig. 4. - (A) Example of the effect of application of the GABA $\mathrm{B}_{\mathrm{B}}$ agonist baclofen. Like GABA, the substance also significantly decreased cell firing and increased the \% of spikes in the response which were within bursts, but never achieved a shift to complete burst "mode" firing. Although in this example a level of $\sim 14 \%$ was achieved with application of $+70 \mathrm{nA}$ of baclofen, a further increase to $+90 \mathrm{nA}$ reduced this value to $\sim 2 \%$. (B) Selected raster plots and PSTHs for control, baclofen $+70 \mathrm{nA}$ and baclofen + $90 \mathrm{nA}$. The reduction in response magnitude is clearly seen. Interestingly, increasing baclofen from 70 to $90 \mathrm{nA}$ resulted in a sudden drop in burst firing. This is the only example we have seen of this phenomenon, but it is clearly observed in the raster plots: spontaneous activity is markedly reduced in both cases but only the "peak" responses, containing bursts, are reduced during 90 nA of baclofen, the remaining response remains clearly visually driven. Bin size is $5 \mathrm{~ms}$. The visual stimulus was a drifting grating of 0.3 cycles $/{ }^{\circ}$ presented at 3 cycles/s.

\subsection{Application of other agents}

Above, we mentioned that ACh application could also affect the tonic/burst ratio and we have examined this in a population of 10 cells. The example given in Fig. 2 illustrates the spectrum of activities we found. Wherever ACh application noticeably increased firing rates, the percentage of spikes found in bursts decreased. However, the purpose of our experiments was to attempt to induce a state of complete burst firing, and so we applied the muscarinic cholinergic antagonists scopolamine ( $\mathrm{n}=4$, selective for $\mathrm{M} 1$ receptor subtypes) and atropine ( $\mathrm{n}=4$, non-selective). In these cases, as illustrated in Fig. 5, we were able to increase the \% of bursts significantly, but again never to a point where no tonic firing occurred. In the example shown, while application of $40 \mathrm{nA}$ of atropine increased the \% of bursts, as the overall response decreased, the maximum \% of spikes in bursts was $16 \%$. A second example is shown below, where the $\%$ of tonic firing is discussed. 


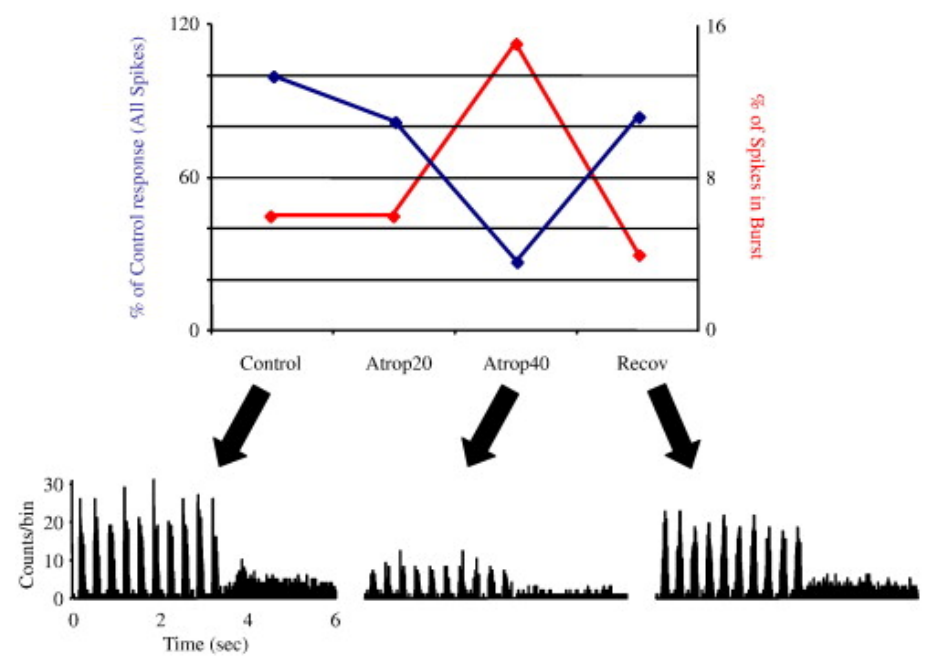

Fig. 5. - Effect of atropine. The effect of anticholinergic drugs was similar in nature to the application of GABA mimetics - a decrease in responsiveness and background firing, coupled with increases in the \% of spikes fired in bursts. Here atropine at $+20 \mathrm{nA}$ significantly lowers the visual response, but the effect on burst firing is not seen until the dose is increased to + $40 \mathrm{nA}$, increasing the \% of burst firing from 6 to $16 \%$. Note that the remaining firing still contains visually elicited structure. The visual stimulus was a drifting grating of 0.3 cycles $/{ }^{\circ}$ presented at 3 cycles $/ \mathrm{s}$.

Decrease in visual responsivity can be produced by application of GABA and GABA-like substances, as described above. However, application of the NOS inhibitor l-NOArg also decreases visual responsiveness (Cudeiro et al., 1994), critically, doing so by an alternative mode of action which may not induce a dose-dependent hyperpolarisation (Cudeiro et al., 1994 and Alexander et al., 2006), Application of l-NOArg in this study clearly reduced visual response magnitude, as illustrated in Fig. 6. In this case, even a reduction by some $32 \%$ of the control value actually slightly decreased the \% of spikes found in bursts from $7.8 \%$ to $5.6 \%$. We tested this on a population of 17 cells, with identical results. Block of NO production reproducibly decreased firing but did not increase the \% of spikes fired in bursts.
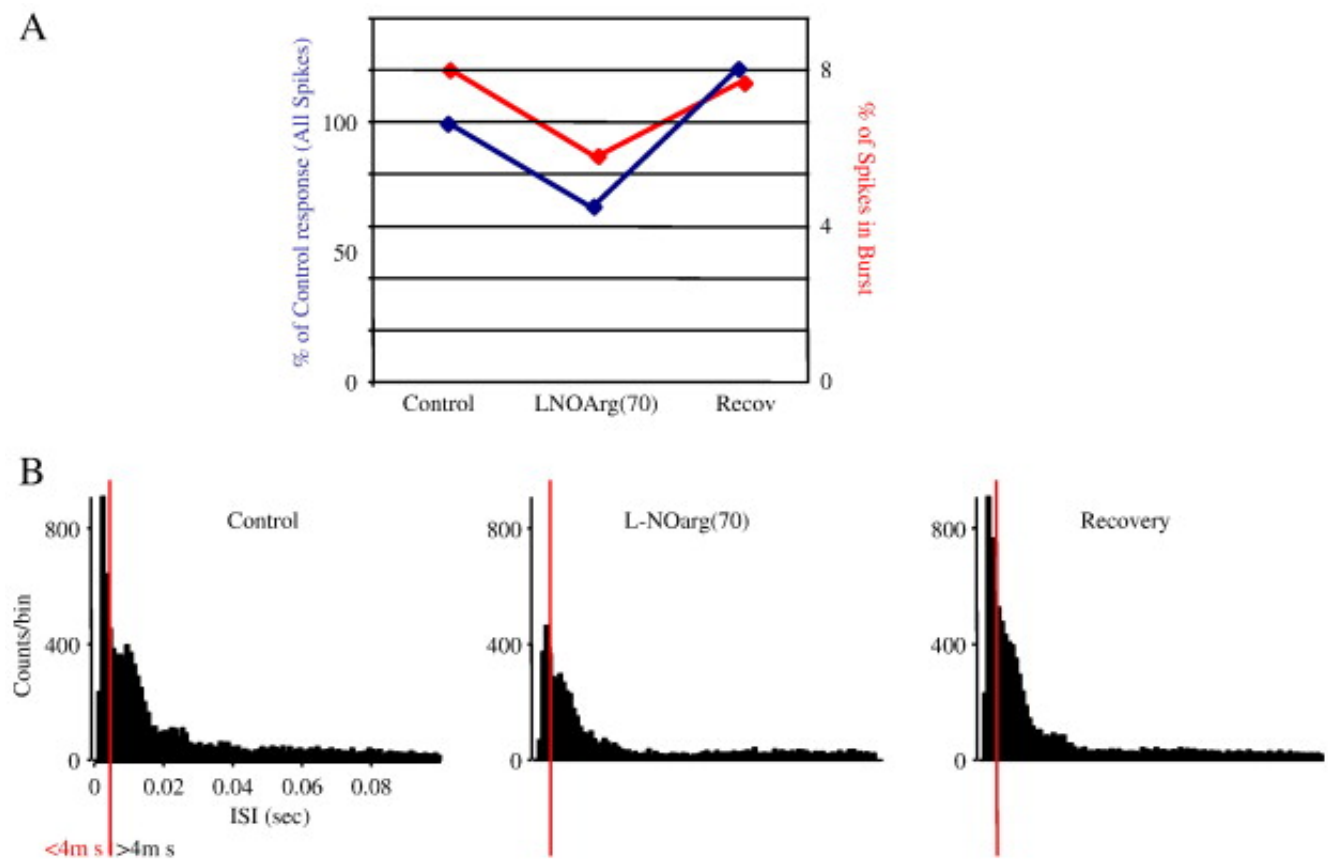

Fig. 6. - Effect of l-NOArg application. (A) As before, the effect of drug application is compared to control and recovery periods, for both all spikes (blue line, left axis) and those spikes included in bursts (red line, right axis). l-NOArg causes a reduction in visually elicited firing, but is coupled with a reduced \% of spikes found in bursts. (B) Shown here are the interspike interval histograms for the 3 conditions. Clearly there is a proportional loss of small values $(<4 \mathrm{~ms}$, left of the red line in each case) compared to those with $>4 \mathrm{~ms}$ (right of the red line in each case) for l-NOArg application, along with an overall reduction. (However, the minimum \% of tonic spikes (see text) also remains the same throughout). 


\subsection{Presence of tonic firing}

We further analysed the data presented above to detect the presence of spikes fired in tonic "mode", both in terms of (i) a minimum value: a $\%$ of the spikes fired which we could be absolutely assured were not fired in bursts, and (ii) their position in the spike trains, relative to bursts. The process is illustrated by the example in Fig. 7. The first step is basically the form of analysis we have carried out for the identification of burst firing, as above. Thus the basic pattern of the responses is shown in Fig. 7A, as before. This ON, X cell was treated with the anti-cholinergic compound scopolamine. The data shown in Fig. 7B directly compares the PSTHS of the control situation against that seen during the most effective dose of scopolamine for evoking bursts, $100 \mathrm{nA}$. It should be noted that this example represents the highest level of burst firing we have seen, in any cell, under any circumstances, and, as such, represents the most extreme case in which we attempt to show burst firing in a single "mode". In the lower part of the figure this PSTH is broken down into its component raster plots, revealing the moment by moment firing of the cell in response to the grating, while $100 \mathrm{nA}$ of scopolamine is applied continuously.

A

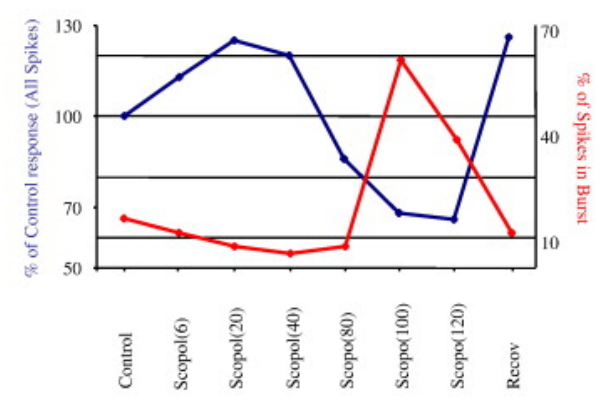

B
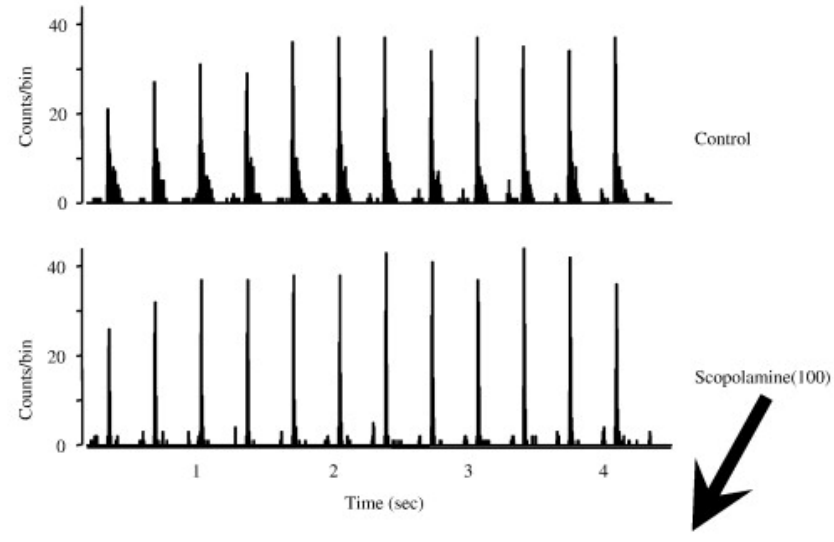

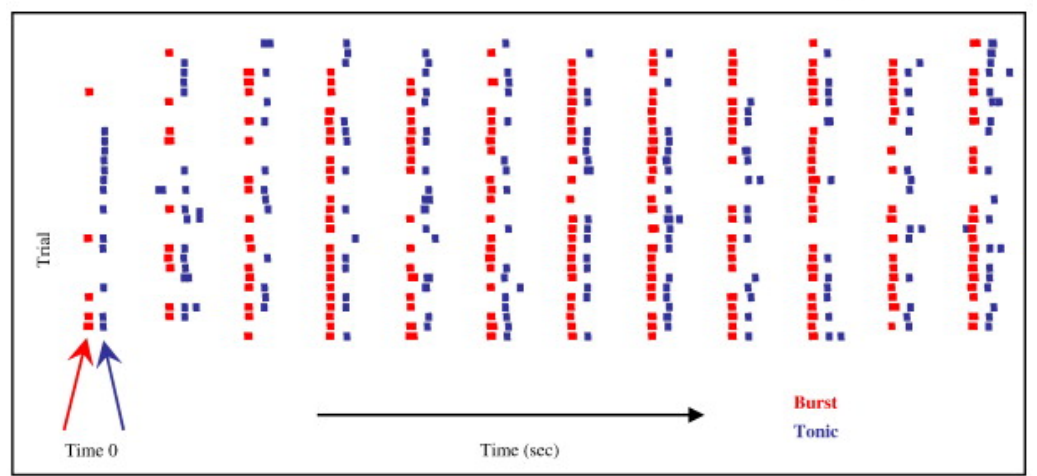

Fig. 7. - The "Minimum \% Tonic Firing”. The example shown here is of an ON, X cell treated with scopolamine, but examples for GABA and GABA agonists show similar findings. (A) As for most of the previous figures, we show the total numbers of spikes elicited (blue line, left axis) versus the spikes occurring in bursts (red line, right axis) — note however, that as scopolamine was increased in "dose" to $+100 \mathrm{nA}$, the \% of spikes fired in bursts reached $70 \%$, the highest figure we have achieved to date. (B) Top — the PSTHs for the control response and the effect of + $100 \mathrm{nA}$ of scopolamine. Lower — the PSTH for the scopolamine response is broken down into its component raster plots. Here we further divide the spikes trains into those spikes which MUST be part of a thalamic burst response (red) and those which must be tonic (blue) - note therefore that the total number of spikes shown may not be $100 \%$ and that, for clarity, we have offset the two spike types on the $x$ axis to reduce overlap. It is absolutely clear that both types 
of firing coexist throughout the duration of the response - there is never a period of either pure tonic or pure burst firing. The visual stimulus was a drifting grating of 0.5 cycles $/{ }^{\circ}$ presented at 3 cycles $/ \mathrm{s}$. 
However, the data have been sorted by two routines; the first, as above, to identify those spikes which appear as a part of a thalamic burst, as defined in our methods section. The same data was then analysed for the presence of tonic spikes ie those preceded by a spike between 4 and $50 \mathrm{~ms}$ and also do not have a spike in the preceding $4 \mathrm{~ms}$. Such spikes are, by definition, fired in tonic mode and cannot be part of a burst. From these data we see that in this case at least $21 \%$ of the spikes fired during the period affected by scopolamine were tonic in nature and about $70 \%$ were in bursts. There are no observable epochs of any significant duration in which the cell fired only in one "mode".

\subsection{Population statistics}

For the population of 18 cells tested with $\mathrm{GABA}_{\mathrm{A}}$ agonists (either GABA or isoguvacine), all cells showed mixed tonic and burst firing whenever firing was present, and the proportions of spikes fired in burst mode changed from $22 \pm 4.8$ (SEM) to $43.2 \pm 9.2 \%$ as the agonist was applied at a level inducing maximum bursting ( $p \leq 0.05$, Wilcoxon test). These remained significant when broken down into GABA $(n=13,24 \pm 4.2(\mathrm{SEM})$ to $46 \pm 6 \% p<0.05)$ and isoguvacine $(n=5,20 \pm 5$ (SEM) to $42 \pm 10 \%$ $p>0.03)$ alone. This also occurred when we used the $\mathrm{GABA}_{\mathrm{B}}$ agonist baclofen $(n=12$; from $13 \pm 4$ to $25 \pm 4.2 \% . p \leq 0.05$ ) or anticholinergic compounds ( $n=9$; from $18.6 \pm 4.9$ to $33.4 \pm 8.8 \%$. $p \leq 0.05$ ). Thus neurons always retained significant levels of tonic firing mixed with bursts throughout their responses. Cells treated with l-NOArg $(n=17)$ decreased their visual responses while retaining, unchanged, the proportions of tonic/burst (proportions of spikes fired in burst mode changed from $20.88 \pm 4.2$ to $18.5 \pm 3.5 \%$; this decrement was not significant).

\section{Discussion}

It is understood that the requirements for a thalamic cell to fire in burst mode is a sufficient degree of hyperpolarisation, for a sufficient period of time to deinactivate the $I_{\mathrm{T}}$ current, allowing incoming excitatory EPSPs to produce a $\mathrm{Ca}^{++}$mediated spike which gives rise to the "normal" $\mathrm{Na}^{+}$spikes ( Jahnsen and Llinas, 1984b), which can easily be recorded extracellularly ( Lo et al., 1991 and Lu et al., 1992). Here, we ( Grieve et al., 2003, Rivadulla et al., 2003, Rivadulla et al., 2005 and de Labra et al., 2007), like many others ( Guido et al., 1992, Guido et al., 1995, Lu et al., 1992 and Ramcharan et al., 2000), have adopted the extracellular technique for the long duration recordings we show here, typically many minutes of continuous recording.

Previous work has suggested that certain stimulus parameters might increase the likelihood of a cell firing in bursts (Guido et al., 1992 and Alitto et al., 2005). It is clear that such parameters, logically, maximise the degree of inhibition present prior to an excitatory input. Further, early literature (Jahnsen and Llinas, 1984b, Crunelli et al., 1988, Soltesz et al., 1989 and McCormick and Pape, 1990) suggested that it would be theoretically possible to manipulate the local milieu around a thalamic dLGN cell and achieve a steady state of continuous burst firing. This has been clearly demonstrated in vitro ( McCormick and Prince, 1987 and McCormick and Prince, 1988). The suggestion is thus: if a cell can be held at a sufficiently hyperpolarised level, repeated depolarising inputs at low temporal frequency should engender only bursts of sodium spikes evoked by an underlying low threshold calcium spike. The implication is that a relatively simple spike-to-spike transition controls the majority of dLGN firing in tonic "mode", which slips into burst "mode" as the strength of this driving is diminished, either by increased local inhibition, or removal of other, non-retinal excitatory inputs. Here, we have applied substances known to mimic the dLGN inhibitory systems, or to disfacilitate dLGN cells by blocking excitatory inputs. The process was combined with visual stimulation at a temporal frequency well within the level shown to be effective in vitro ( McCormick and Feeser, 1990). Local blockade of NOS produced a reduction in firing rate without a concomitant shift in bursting. We believe that this can be explained most simply by previous findings with regard to NO activity in the thalamus, which have been shown to be mediated through an interaction with the NMDA receptor ( Cudeiro et al., 1994 and Alexander et al., 2006, but also see Pape and Mager, 1992). Blockade of NOS would therefore result in a decrease in firing as activity evoked through NMDA receptors was removed. However, the non-linear nature of this receptor would mean that the cell would be unlikely to be hyperpolarised below the active range of the NMDA receptor, with almost no activity at $-60 \mathrm{mV}$ ( Mayer et al., 1984 and Nowak et al., 1984). Application of GABA, subtype specific GABA agonists and modulators of the cholinergic system produced a different result.

These show quite clearly that although the proportion of spikes fired in bursts increases as cells become more inhibited, it is not possible to reach a steady state of burst firing alone under any of the conditions we have generated. Instead, continued significant elements of tonic firing appear throughout the recordings, even when firing rates approach zero. This view is seen not only if we used the accepted 
definition of a burst, but also if we apply the stringent criterion we have now devised to detect the presence of tonic firing. Viewed simplistically, application of GABA and GABA-like agonists are known to hyperpolarise neurons, through well-defined ionic mechanisms, which reduce cell firing, spontaneous and visually elicited. Similarly, the action of compounds manipulating the cholinergic system, are also known, by withdrawal of an excitatory input, to effect a hyperpolarising action on dLGN cells. It is therefore not at all surprising that the rate of firing was reduced. However, despite reducing firing levels to near zero in many cases, and to zero in a significant number of cases, we did not find evidence of prolonged episodes of burst firing indicative of a switch of "state". There was a complex mix of tonic and burst firing seen at all levels, with at best a weak correlation with the decrease in firing and the proportion of spikes fired in bursts. Our findings therefore reinforce the view (McCarley et al., 1983, Esguerra and Sur, 1993, Ramcharan et al., 2000 and Sherman and Guillery, 2002) that control of bursting, and rhythmic firing, in thalamus is more complex than originally suggested. Indeed, other factors have now been shown to increase the complexity of thalamic circuitry, including elements such as extra synaptic $\mathrm{GABA}_{\mathrm{A}}$ receptors (Cope et al., 2005), presynaptic GABA receptors (Chen and Regehr 2003), and multiple routes of information flow though intrinsic inhibitory cells via distal dendrites (Cox and Sherman, 2000). More recently, Wang et al. (2006) have demonstrated that the extent of burst firing may be under the control of corticofugal feedback, tightly coupled to the nature of the visually elicited input from layer 6 cells, probably as a result of changes in the gain of intrinsic (local or PGN) inhibitory cells. Most notably however, these authors reported that changes in burst/tonic ratios were "shifts in the ratio, not a complete switch between firing modes" (Wang et al., 2007, page 1332), just as we report here.

Non-visual modulatory influences may also operate through direct action on LGN relay cell and actions on inhibitory interneurons. Compounds acting on the cholinergic system have well recognized excitatory effects on relay cells (Sillito et al., 1983 and McCormick and Prince, 1987), and inhibitory effects on local, GABAergic (!) inhibitory interneurons (McCormick and Pape 1988) and can also act upon nicotinic cholinergic receptors (for example, Zhu and Uhlrich 1997). The in vivo complexity far exceeds the simplistic notion of an "awake" brain slice, and our data clearly supports the earlier suggestions ( McCarley et al., 1983, Esguerra and Sur, 1993, Ramcharan et al., 2000 and Sherman and Guillery, 2002) and the suggestions of Wolfart et al. (2005), who, using a guinea pig thalamic slice subject to dynamic-clamp conclude that spontaneous noise fluctuations might be sufficient to prevent thalamic cells from "settling" into one state or another.

It has been estimated that the retinal input to cells in the dLGN may comprise as little as $7 \%$ of the total synaptic weight (Van Horn et al., 2000). With the low temporal frequency visual input we have utilised here, we could reasonably expect to induce, and maintain, burst mode firing of a significant proportion of cells recorded, if we were able to induce and maintain a stable level of membrane hyperpolarisation, (at least if conditions seen in brain slice preparations could be mimicked). This is clearly not possible in our anaesthetised cat preparation. It is highly likely that, even during the state of anaesthesia typical of visual experiments, there is a sufficient rich synaptic barrage on any given cell to maintain a mixture of burst and tonic firing patterns, even when exogenous application of substances such as GABA bring the firing of the cell to near zero. Thus in the anaesthetised animal we see continuous shifting of firing from burst to tonic and reverse over very short time periods, and this may be representative of the "state" of the thalamus in vivo - no distinct periods of burst or tonic firing, but a continuously shifting position along a continuum which falls short of either "mode".

\section{Experimental procedures}

Experiments were carried out on 5 adult cats of either sex. Animals were anaesthetised with ketamine and xylazine for induction and maintained on halothane in $\mathrm{N}_{2} \mathrm{O}$ and $\mathrm{O}_{2}(0.5-1 \%$ in 50:50 gases), and paralysed with gallamine triethiodode $(10 \mathrm{mg} / \mathrm{kg} / \mathrm{h})$. EEG and ECG were monitored continuously and end-tidal $\mathrm{CO}_{2}$ was maintained between 3.8 and $4.2 \%$. Any changes commensurate with change in anaesthetic status were countered by changes in the administered halothane level. All procedures were carried out to the standards approved by the Spanish Physiological Society and the UK Home Office. Other details have been previously reported (Cudeiro et al., 2000 and Rivadulla et al., 2003).

Single units were recorded in the dLGN using multi-barrelled micropipettes filled with a selection of the following drug solutions: $\mathrm{NaCl}(3 \mathrm{M}$, for recording), ACh chloride (1 M, $\mathrm{pH}$ 5), atropine sulphate (0.1 M, pH 4.5), baclofen (50 mM, pH 4), gamma-aminobutyric acid (GABA, 0.5 M, pH 3.5), isoguvacine (0.1 M, pH 4.5), l-nitro-arginine (l-NOArg; $10 \mathrm{mM}, \mathrm{pH6} .0$ ), sodium chloride (150 mM) and scopolamine $(0.1 \mathrm{M}, \mathrm{pH} 4.5)$. All drugs were retained with current levels between 5 and $25 \mathrm{nA}$ of appropriate polarity. Ejection current levels are given in the text.

Visual stimuli comprised drifting sinusoidal gratings. The temporal frequency was selected to achieve intermittent visual stimulation coupled with periods of inhibition or disfacilitation of sufficient duration to 
permit the de-inactivation of $I_{\mathrm{T}}$, a prerequisite for the production of burst firing. Stimuli were delivered monocularly to the appropriate eye, under computer control. Receptive fields were characterised as X or $\mathrm{Y}$ according to the linearity of their visual response, size of field (relative to location of area centralis), and responses to higher temporal frequency. Gratings were optimised in terms of contrast (routinely $60 \%$ ), spatial frequency for each cell under test, and the temporal frequency was selected to be within the range which we expected to be effective in inducing burst firing, but also such that the cell responded robustly in the control conditions, thereby producing sufficient spikes to analyse effectively. In all cases, we used stimuli which were within the accepted "normal" range for LGN cells, as previously published throughout the historic literature. Stimuli were presented full-field on a monitor occupying $32 \times 24^{\circ}$ of visual space, and all receptive fields were located within $12^{\circ}$ of the area centralis. Stimuli were presented and responses recorded using the Leonardo system (Lohmann Research Equipment, Germany), coupled to a Plexon system (Plexon Inc., USA). Off-line analysis was carried out using NEX (Plexon Inc., USA) and Matlab (The MathWorks, UK). Visual responses were separated into spikes which were considered to be "tonic" and those in "bursts" and counted, as we have previously described ( Rivadulla et al., 2003). Basically, bursts comprised 3 or more spikes separated by interspike intervals of less than $4 \mathrm{~ms}$, which were preceded by a silent period of at least $50 \mathrm{~ms}$. Except as described below, all other spikes were classified as tonic. Other authors have used similar methods ( Guido et al., 1992, Guido et al., 1995, Guido and Weyand, 1995, Reinagel et al., 1999 and Weyand et al., 2001) except that they used two spikes as the criteria for being considered as a burst. The main risk of classifying spikes as burst or tonic using extracellular recordings is that high frequency tonic spikes (HT burst, Guido et al., 1992; pseudoburst, Swadlow and Gusev, 2001) can be considered as being in LT bursts. This is part of the unfortunate but universal problem for the identification of any burst in extracellular recording. Because of this, we devised a further analysis to calculate the "minimum number" of tonic spikes in any spike train by exclusion of all spikes whose position in the spike train meant that they had the potential to be evoked by the low threshold calcium spike which underlies a burst. This meant that only spikes which were (a) preceded by another spike in the time interval of $>4$ but $<50 \mathrm{~ms}$ and also (b) did not have a spike in the preceding 4 ms were included. Such spikes could therefore neither be in the middle of a burst (where the preceding interval must be less than $4 \mathrm{~ms}$ ) nor be the first spike in a burst (where the preceding interval would have to exceed $50 \mathrm{~ms}$. The value we produce is not a true measure of tonic firing, but a measure of the minimum level of tonic firing and an indication of the continued presence of tonic firing. Some of these data have been presented to the UK and Spanish Physiological Societies (Joint meeting, Grieve et al., 2003).

Acknowledgments. Supported by MEC (BFU2005-00502) and Xunta de Galicia (Consellería de Educación-2007/000140-0), Spain.

\section{REFERENCES}

Alexander et al., 2006. G.M. Alexander, N.C. Kurukulasuriya, J. Mu, D.W. Godwin. Cortical feedback to the thalamus is selectively enhanced by nitric oxide. Neuroscience, 142 (2006), pp. 223-234

Alitto et al., 2005. H.J. Alitto, T.G. Weyand, W.M. Usrey. Distinct properties of stimulus evoked bursts in the lateral geniculate nucleus. J. Neurosci., 25 (2005), pp. 514-523

Chen and Regehr, 2003. C. Chen, W.G. Regehr. Presynaptic modulation of the retinogeniculate synapse. Neurosci., 23 (2003), pp. 3130-3135

Cope et al., 2005. D.W. Cope, S.W. Hughes, V. Crunelli. GABA ${ }_{A}$ receptor-mediated tonic inhibition in thalamic neurons. J. Neurosci., 25 (2005), pp. 11553-11563

Cox and Sherman, 2000. C.L. Cox, S.M. Sherman. Control of dendritic outputs of inhibitory interneurons in the lateral geniculate nucleus. Neuron, 27 (2000), pp. 597-610

Crunelli et al., 1988. V. Crunelli, M. Haby, D. Jassik-Gerschenfeld, N. Leresche, M. Pirchio. $\mathrm{Cl}^{-}$and $\mathrm{K}^{+}$-dependent inhibitory post-synaptic potentials evoked by interneurones of the rat lateral geniculate nucleus. J. Physiol., 399 (1988), pp. 153-176

Crunelli et al., 2005. V. Crunelli, T.I. Tóth, D.W. Cope, K. Blethyn, S.W. Hughes. The 'window' T-type calcium current in brain dynamics of different behavioural states. J. Physiol., 562 (2005), pp. 121-129

Cudeiro et al., 1994. J. Cudeiro, K.L. Grieve, C. Rivadulla, R. Rodriguez, S. Martinez-Conde, C. Acuna. The role of nitric oxide in the transformation of visual information within the dorsal lateral geniculate nucleus of the cat. Neuropharmacology, 33 (1994), pp. 1413-1418

Cudeiro et al., 2000. J. Cudeiro, C. Rivadulla, K.L. Grieve. Visual response augmentation in cat (and macaque) LGN: potentiation by corticofugally mediated gain control in the temporal domain. Eur. J. Neurosci., 12 (2000), pp. 1135-1144

de Labra et al., 2007. C. de Labra, C. Rivadulla, K. Grieve, J. Marino, N. Espinosa, J. Cudeiro. Changes in visual responses in the feline dLGN: selective thalamic suppression induced by transcranial magnetic stimulation of V1. Cereb. Cortex, 17 (2007), pp. 1376-1385 
Esguerra and Sur, 1993. M. Esguerra, M. Sur. Spike trains and signaling modes of neurons in the ferret lateral geniculate nucleus. Exp. Brain Res., 96 (1993), pp. 273-286

Glen and Steriade, 1982. L.L. Glen, M. Steriade. Discharge rate and excitability of cortically projecting intralaminar thalamic neurons during waking and sleep states. J. Neurosci., 2 (1982), pp. 1387-1404

Grieve et al., 2003. K.L. Grieve, C. Rivadulla, L. Martinez, J. Cudeiro. Neuromodulation of thalamic response modes in the lateral geniculate nucleus of the cat. J. Physiol., 548 (2003), p. P104

Guido and Weyand, 1995. W. Guido, T. Weyand. Burst responses in thalamic relay cells of the awake behaving cat. J. Neurophysiol., 74 (1995), pp. 1782-1786

Guido et al., 1992. W. Guido, S.M. Lu, S.M. Sherman. Relative contributions of burst and tonic responses to the receptive field properties of lateral geniculate neurons in the cat. J. Neurophysiol., 68 (1992), pp. 2199-2211

Guido et al., 1995. W. Guido, S.M. Lu, J.W. Vaughan, D.W. Godwin, S.M. Sherman. Receiver operating characteristic (ROC) analysis of neurons in the cat's lateral geniculate nucleus during tonic and burst response mode. Vis. Neurosci., 12 (1995), pp. 723-741

Jahnsen and Llinas, 1984a. H. Jahnsen, R. Llinas. Electrophysiological properties of guinea-pig thalamic neurones: an in vitro study. J. Physiol., 349 (1984), pp. 205-226

Jahnsen and Llinas, 1984b. H. Jahnsen, R. Llinas. Ionic basis for the electroresponsiveness and oscillatory properties of guinea-pig thalamic neurones in vitro. J. Physiol., 349 (1984), pp. 227-247

Llinas and Steriade, 2006. R. Llinas, M. Steriade. Bursting of thalamic neurons and states of vigilance. J. Neurophysiol., 95 (2006), pp. 3297-3308

Lo et al., 1991. F.S. Lo, S.M. Lu, S.M. Sherman. Intracellular and extracellular in vivo recording of different response modes for relay cells of the cat's lateral geniculate nucleus. Exp. Brain Res., 83 (1991), pp. 317-328

Lu et al., 1992. S.M. Lu, W. Guido, S.M. Sherman. Effects of membrane voltage on receptive field properties of lateral geniculate neurons in the cat: contributions of the low-threshold $\mathrm{Ca}^{2+}$ conductance. J. Neurophysiol., 68 (1992), pp. 2185-2198

Mayer et al., 1984. M.L. Mayer, G.L. Westbrook, P.B. Guthrie. Voltage-dependent block by $\mathrm{Mg}^{2+}$ of NMDA responses in spinal cord neurons. Nature, 309 (1984), pp. 261-263

McCarley et al., 1983. R.W. McCarley, O. Benoit, G. Barrionuevo. Lateral geniculate nucleus unitary discharge in sleep and waking: state- and rate-specific aspects. J. Neurophysiol., 50 (1983), pp. 798-818

McCormick and Bal, 1994. D.A. McCormick, T. Bal. Sensory gating mechanisms of the thalamus. Curr. Opin. Neurobiol., 4 (1994), pp. 550-556

McCormick and Feeser, 1990. D.A. McCormick, H.R. Feeser. Functional implications of burst firing and single spike activity in lateral geniculate nucleus neurons. Neuroscience, 39 (1990), pp. 337-388

McCormick and Pape, 1988. D.A. McCormick, H.C. Pape. Acetylcholine inhibits identified interneurons in the cat lateral geniculate nucleus. Nature, 334 (1988), pp. 246-248

McCormick and Pape, 1990. D.A. McCormick, H.C. Pape. Noradrenergic and serotonergic modulation of a hyperpolarization-activated cation current in thalamic relay neurons. J. Physiol., 431 (1990), pp. 319-342

McCormick and Prince, 1987. D.A. McCormick, D.A. Prince. Actions of acetylcholine in the guinea-pig and cat medial and lateral geniculate nuclei, in vitro. J. Physiol., 392 (1987), pp. 47-65

McCormick and Prince, 1988. D.A. McCormick, D.A. Prince. Noradrenergic modulation of firing pattern in guinea pig and cat thalamic neurons, in vitro. J. Neurophysiol., 59 (1988), pp. 978-996

Nowak et al., 1984. L. Nowak, P. Bregestovski, P. Ascher, A. Herbet, A. Prochiantz. Magnesium gates glutamateactivated channels in mouse central neurons. Nature, 307 (1984), pp. 462-465

Pape and Mager, 1992. H.C. Pape, R. Mager. Nitric oxide controls oscillatory activity in thalamocortical neurons. Neuron, 9 (1992), pp. 441-448

Pape and McCormick, 1995. H.C. Pape, D.A. McCormick. Electrophysiological and pharmacological properties of interneurons in the cat dorsal lateral geniculate nucleus. Neuroscience, 68 (1995), pp. 1105-1125

Ramcharan et al., 2000. E.J. Ramcharan, J.W. Gnadt, S.M. Sherman. Burst and tonic firing in thalamic cells of unanaesthetized, behaving monkeys. Vis. Neurosci., 17 (2000), pp. 55-62

Reinagel et al., 1999. P. Reinagel, D. Godwin, S.M. Sherman, C. Koch. Encoding of visual information by LGN bursts. J. Neurophysiol., 81 (1999), pp. 2558-2569

Rivadulla et al., 2003. C. Rivadulla, L. Martinez, K.L. Grieve, J. Cudeiro. Receptive field structure of burst and tonic firing in feline lateral geniculate nucleus. J. Physiol., 553 (2003), p. 610

Rivadulla et al., 2005. C. Rivadulla, K.L. Grieve, C. De Labra, J. Cudeiro. Multiple systems modulating firing mode in the LGN of the cat. SFN Abstracts, 35 (2005), p. 5063

Sherman, 2005. S.M. Sherman. Thalamic relays and cortical functioning. Prog. Brain Res., 149 (2005), pp. 107-126

Sherman and Guillery, 2002. S.M. Sherman, R.W. Guillery. The role of the thalamus in the flow of information to the cortex. Philos. Trans. R. Soc. Lond., B Biol. Sci., 357 (2002), pp. 1695-1708

Sillito et al., 1983. A.M. Sillito, J.A. Kemp, N. Berardi. The cholinergic influence on the function of the cat dorsal lateral geniculate nucleus (dLGN). Brain Res., 280 (1983), pp. 299-307

Soltesz et al., 1989. I. Soltesz, S. Lightowler, N. Leresche, V. Crunnelli. Optic tract stimulation evokes GABA but $\mathrm{GABA}_{\mathrm{B}}$ IPSPs in the rat ventral lateral geniculate nucleus. Brain res., 479 (1989), pp. 49-55

Swadlow and Gusev, 2001. H.A. Swadlow, A.G. Gusev. The impact of 'bursting' thalamic impulses at a neocortical synapse. Nat. Neurosci., 4 (2001), pp. 402-408

Van Horn et al., 2000. S.C. Van Horn, A. Erisir, S.M. Sherman. The relative distribution of synapses in the Alaminae of the lateral geniculate nucleus of the cat. J. Comp. Neurol., 416 (2000), pp. 509-520

Wang et al., 2006. W. Wang, H.E. Jones, I.M. Andolina, T.E. Salt, A.M. Sillito. Functional alignment of feedback effects from visual cortex to thalamus. Nat. Neurosci., 9 (2006), pp. 1330-1336 
Wang et al., 2007. X. Wang, Y. Wei, V. Vaingankar, Q. Wang, K. Koepsell, F.T. Sommer, J.A. Hirsh. Feedforward excitation and inhibition evoke dual modes of firing in the cat's visual thalamus during naturalistic viewing. Neuron, 55 (2007), pp. 465-478

Weyand et al., 2001. T.G. Weyand, M. Boudreaux, W. Guido. Burst and tonic response modes in thalamic neurons during sleep and wakefulness. J. Neurophysiol., 85 (2001), pp. 1107-1118

Wolfart et al., 2005. J. Wolfart, D. Debay, G. Le Masson, A. Destexhe, T. Bal. Synaptic background activity controls spike transfer from thalamus to cortex. Nat. Neurosci., 8 (2005), pp. 1760-1767

Zhu and Uhlrich, 1997. J.J Zhu, D.J. Uhlrich. Nicotinic receptor-mediated responses in relay cells and interneurons in the rat lateral geniculate nucleus. Neuroscience, 80 (1997), pp. 191-202 